\title{
Advances and Challenges for Scalable Cloud-based Infrastructure For Building Data Analysis and Simulation
}

\author{
Thibaud Nesztler, Michael Georgescu \\ Ecorithm, Santa Barbara, United States of America \\ tnesztler@ecorithm.com, michael.georgescu@gmail.com
}

\begin{abstract}
In the commercial building sector, retrocommissioned and new constructions alike are implementing the use of highly integrated and connected Building Information Systems (BISs) to use fewer resources, improve occupant health and productivity and reduce life-cycle costs. Even if programs such as Leadership in Energy and Environmental Design (LEED) are becoming more and more popular, single factors such as horizontal integration at the portfolio level can drive this change leading to an increase in simulation and forecasting demand.
\end{abstract}

BISs generate large amounts of data from various sources such as control networks or utilities. This data is critical in applications such as continuous commissioning through Automated Fault Detection and Diagnostics (AFDD) or predictive analytics. Moreover, depending on the analysis constraints such as computational maximum runtime or overall cost as well as results' availability and presentation, current techniques can prove to be challenging to use or integrate.

This paper aims to introduce to the building performance simulation's space, techniques from other fields such as computer science and data analytics to help improve quality, reproducibility, scalability of workflows used by this industry and research community. Cloud computing and open source technologies described in this paper can help answer many of the aforementioned challenges. When dealing with big datasets, file formats such as Comma Separated Values (CSV) offer little compression resulting in large files creating unnecessary costs and increased query complexity. File formats used by distributed query engines such as Parquet can offer 98\% size reduction while being easily queryable using the Structured Query Language (SQL) at the expense of time compared to relational databases which can be subsequently used as a caching mechanism.

Containers, often used for cloud-based applications, can also be used for simulation offering scalable, reproducible and hardware agnostic environment to deploy large scale analysis at the expense of a little performance overhead, less than full-fledged Virtual Machines (VMs). Moreover using Infrastructure-asa-Service (IaaS) can allow significant cost reductions by paying only for what is needed in terms of compute and/or memory. For example, running AFDD on $200+$ buildings can cost on the order of less than a US dollar per day which is equivalent to a quarter the cost of running a local server. Web technologies such as Javascript-based User Interfaces (UIs) can as well be used to distribute and access simulation results as well as managing the simulation engine altogether remotely. This enables collaboration of international team members as well as deployment of analytics to international or remote clients.

\section{Introduction}

Within the commercial building space, significant opportunities to reduce energy consumption lie in the efficient operation of Heating, Ventilation and AirConditioning (HVAC) systems. Poorly maintained and improperly controlled HVAC equipment are responsible for up to $22 \%$ of the energy use of a building's HVAC system (Basarkar et al. (2011)). In the United States of America, commercial buildings represent $18.6 \%$ of the total primary energy consumption (U.S. Department of Energy (2012)). Thus, measures to improve the operation of building HVAC systems can have significant national, and global, impact.

A cross-section of studies in literature have examined the efficacy of HVAC operations focusing on the discrepancy between expectations of performance at the design stage versus actual observed performance. Numerous causes have been observed including operational problems such as improper equipment installation, equipment degradation, sensor failures, or control logic problems (Djuric and Novakovic (2009), Wang et al. (2013)). In particular, the work of Torcellini et al. (2006) contains several prominently referenced case studies revolving around underperformance of net zero-energy buildings. A conclusion echoed throughout these studies is that building underperformance is not limited to buildings that are old or new, or buildings designed with energy efficiency measures implemented throughout the design 
process. These industrial case studies conclude that underperformance is caused by insufficient monitoring and underdeveloped analysis of the systems and equipment managed where a lack of availability of building operational data is a key determinant of whether a building will meet its expected energy efficiency goals.

Traditionally, building performance has been the responsibility of an organization's facilities management team where underperformance is determined through energy audits. These audits, on a preliminary level, include building walkthroughs and review of facility utility bills and scale up to detailed energy analysis, building life cycle cost analysis, and determination of more capital-intensive improvements through retro-commissioning (ASHRAE (2018)). A growing requirement within organizations however is to develop corporate level sustainability planning which introduces new stakeholders and objectives into the facilities management realm. Although these changes are positive in the long term, they may also be counterproductive, in the short term, as the addition of stakeholders and objectives can introduce complicated workflows and data silos. Energy audits are limited in this context as they are time consuming and effectively applying a single-building-at-atime type workflow to a portfolio level. Although audits can be effective, particularly in situations where equipment retro commissioning is needed, it is a costly first pass at analysis. A need exists for systems that can work continuously at a portfolio level and are extensible thereby enabling the development of integrations and analysis allowing stakeholders to interoperate.

A unifying thread between stakeholders is shared access to data. In modern commercial buildings, such information sources are available through the Building Automation System (BAS) which, when integrated into complex sensor networks, generate large amounts of data. However, until recently, this data was only used for sporadic problem-solving. Recognizing the potential of the BAS as a data source, practitioners have begun to develop techniques which comprehensively utilize this data in ongoing operational tasks. Depending on the frequency that data is collected, solutions fall into one of two categories: dedicated edge deployments that are on premises or cloud-based. Edge based solutions include projects such as the Building Controls Virtual Test Bed (Wetter (2011)) which operate building equipment through model predictive control via co-simulation of a building's systems in order to determine a forecast of some optimal operational profile. Alternatively, in a transition away from costly occasional energy audits, cloud-based Building Information Systems (BISs) have begun to surface which augment the BAS stack by adding a continuous and ondemand online analytics / informational layer. These systems are invaluable as they enable Fault Detection and Diagnostics (FDD), energy monitoring, continuous commissioning, and other supportive tasks in the building management process.

In this work, the requirements and challenges of developing building information analysis platforms are examined. A case-study is evaluated where monitoring and analysis of building data is presented utilizing simulations running on cloud-based systems. The software stack utilizes a variety of open-source database / data warehouse technologies for scalable storage with performant querying of raw data and aggregates. Additionally, open-source computing frameworks enable simulation environments which can be executed on-demand and at low per-unit compute cost. These tools enable an intuitive development environment for creating maintainable applications. When combined with a web application interface, the confluence of these technologies allow for a scalable BIS that can integrate data from multiple building portfolios and is extensible to support new data sources and applications. The goal of this paper is to present usage patterns to motivate more widespread adoption of these tools within the community of building analysts.

\section{Background}

A BIS generates and stores multiple types of data ranging from HVAC or lighting sensor, energy meter, occupancy or security video data to tenant billing, utility bills, building schedules or mechanical drawings. Facilities need easy access to this data for multiple operations such as maintenance, management, billing or security. However, the personnel can't analyze in its entirety, the raw dataset generated by a BIS. Building operators will focus their analysis efforts on only key areas. For example, the biggest inefficiencies and largest savings opportunities are often located at the central plant level (Air Handling Units (AHUs), chillers, cooling towers, boilers, etc.). Modern computers through their repetitive task handling capabilities and compute power can help reduce various building inefficiencies via a combination of building simulation, continuous commissioning and Automated Fault Detection and Diagnostics (AFDD).

A wide variety of analysis and simulations can be decomposed into at least three tasks: raw data management, computation and visualization. They are at their core, data related jobs. Other fields, in particular software engineering, have been looking at how to solve these tasks as well and overcoming their inherent issues. In this field, a concept known as clean architecture (Martin (2017)) is used to improve the efficiency of various systems. Figure 1 shows the clean architecture concept as well as the main elements found in each layer. Layers are independent from each other and the dependencies between layers can only move inwards. Moreover the inner layers 
close to the core are generic and unlikely to be modified while the outer layers are very specific and prone to changes. By using software as inspiration for solving the challenges of building simulation, it could be possible to improve buildings' efficiency.



Figure 1: Clean architecture concept. Parallelism between software architecture and building simulation can be drawn to increase buildings' efficiency through continuous commissioning, building simulation and Automated Fault Detection and Diagnostics (AFDD).

Multiple people can be in charge of maintaining each data set as well as owning them. This results in data being siloed within a company's service or external ones. Data silos can create inefficiencies in how data is shared across members often due to the tools used such as email, public cloud-storage solutions, thumb-drives or even paper for in-person meetings. They also have the tendency to create heterogeneous storage solution both in terms of data formats or accessibility. Finally, data can be at a security risk due to where and how it is stored, accessed and shared. Using Application Programming Interfaces (APIs) and/or standard data formats can improve the efficiency and security of such system when accessing or sharing data between applications, infrastructures or devices remotely.

Data generated by the BAS is often stored on-premise at each building. Institutions which have to deal with numerous buildings have nonetheless started centralizing their data at a specific location for remote building control and operations. In these cases, proprietary software is often used to collect, store and display data making interoperability with other software architectures often tied to hefty licensing fees for drivers. However, some international standards and data collection tools have emerged easing this process such as the BACnet protocol (ASHRAE (2016)) in 2003, the VOLTTRON platform (VOLTTRON (2019)) or Project Haystack (Project Haystack Corporation (2019)) both in 2014. Moreover, using opensource data storage solutions in combination with a centralized data store (at the same location or within the same network) can enhance the interoperability with other companies and software architectures alongside tightening its security.

Analysis of this accumulated data can be added to the BIS providing another layer of insight into a specific building or portfolio. The challenges are more significant when you have to provide results for an entire portfolio, since buildings within the same institution can have many different designs and operation requirements. This is especially true in the case of commercial real-estate corporations where selling, buying and constructing new buildings and having a wide variety of tenants is common practice. Simulation and analysis need to be agnostic of buildings' equipment in order to scale with the portfolio easily. This also applies to the storage solution which needs to handle fluctuations in the portfolio's size and in the types and sources of data being generated.

When looking at continuous commissioning and/or AFDD, simulation runtime over an entire portfolio can exceed tens of minutes even hours. This creates an issue where a lot of time is spent waiting on results that might not be satisfactory, especially during the analysis tuning phase. To reduce it, scaling the processing power can prove very costly on-premise due to the requirements of server-grade components and installations. Moreover, depending on the tools used, it cannot be done cost-effectively due to software license limitations. Cloud-based solutions with Open-Source Software (OSS) can offer a cost and performance advantage over on-premise installations. Additionally, using the cloud and its technologies can boost productivity and security through near $24 / 7$ availability while tools such as containers allow easy deployment of new features and updates.

Finally, building inefficiencies could not be solved without proper communication between the appropriate parties. The need to share meaningful information efficiently becomes prominent. Internet and the technologies that resulted from its use as a fast, efficient and universal means of communication can help solve this problem. Researchers and data scientists can benefits from these cloud and Web technologies towards a clean research initiative encouraging a collaborative work environment. Their work can be shared online alongside visualizations and reproduced easily, increasing transparency and validation by other scientists. 


\section{Cloud and Open Source Technologies}

\section{Cloud Technologies}

Cloud computing is defined as a paradigm for enabling network access to a scalable and elastic pool of shareable physical or virtual resources with selfservice provisioning and administration on-demand (ISO (2014)). Cloud computing services are delivered using 3 different models or capability types:

- Software-as-a-Service (SaaS) or Application Capability Type (ACT)

- Platform-as-a-Service (PaaS) or Platform Capability Type (PCT)

- Infrastructure-as-a-Service (IaaS) or Infrastructure Capability Type (ICT)

We are going to focus our interests on the PaaS model which allows the deployment of applications and configuration settings to the cloud infrastructure deployed by providers which can be public such as Google Cloud Platform, Amazon Web Services, Microsoft Azure, Salesforce's Heroku or private.

Building simulations and information systems can be developed locally and then deployed to the cloud. Two competing approaches allow such use case as illustrated in Figure 2.
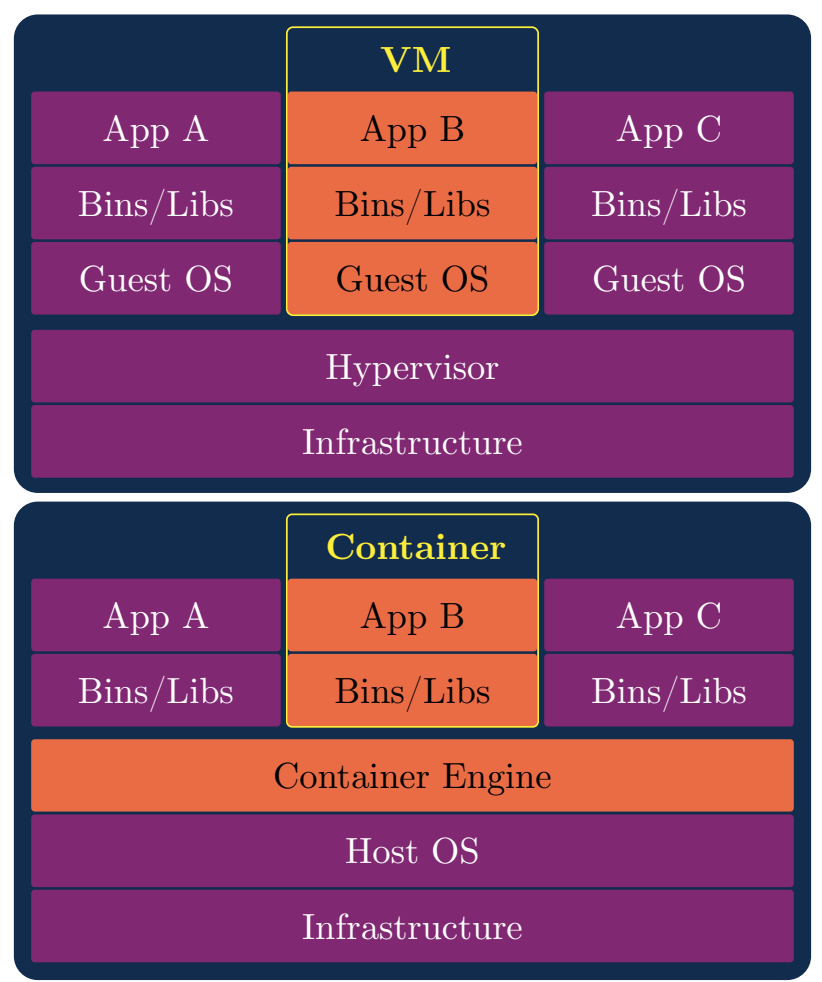

Figure 2: Two competing approaches towards deploying workflows such as simulation in the cloud while offering local development capabilities: containers versus Virtual Machines (VMs). The application requires in both cases its dependencies (binaries and libraries). The differences lie within the interaction of the application and the resources such as the Operating System (OS) and infrastructure.
The first and most common is to use Virtual Machines (VMs) which are environments created by the simulation of the software and/or hardware upon which other software runs (NIST (2011)). This allows the deployment of workflows already present on Personal Computers (PCs) to the cloud. The second one is using containers, a method for packaging and securely running an application within an application virtualization environment. By inferring constraints, containers are lighter, more efficient and easier to develop and deploy than VMs. Container technology have been gaining traction over the past decade with open-source implementations such as Docker or the Open Container Initiative (OCI) and availability on several Cloud Service Providers (CSPs).

With a Docker implementation, the application's code and its dependencies are packaged into what is called an image. Images are read-only, often based on other images such as "ubuntu" and then customized. An image can be resumed to a set of instructions to build it - called a Dockerfile. Images can be uploaded to public or private registries as well, making them easy to share with others.

A container is an instance of an image so multiple copies of an image can be run at the same time, with different environment variables, making them suitable for scalable software stacks or infrastructure based on resources or demand. They could be used for sensitivity analysis for example.

Cloud computing, with its pay-as-you-go model can offer great cost benefits due to its monitoring and scalability capabilities. Since most services are billed hourly or monthly, running simulation in the cloud can become cost and time efficient compared to onpremise simulation when they require variable compute power. CSPs offer server-grade hardware which enables a variety of instance types that can be tailored for variable use-cases such as compute, memory or graphics-accelerated workloads. For example a practitioner can get a memory optimized instance delivering large amounts of memory while not paying for unused compute power. As such, it has been shown that running large-scale (over 1 million) EnergyPlusbased building simulations on cloud instances versus on-premise can yield runtime reduction of $95 \%$ for a cost less than $2500 \$$ (Richman et al. (2014)).

\section{Open Source Technologies}

Open-Source Software (OSS) is software distributed under a license which at minima gives free access to its source code and allows modifications and redistribution without additional permissions (Open Source Initiative (2019)). OSS has been growing exponentially since the 1990s (Deshpande and Riehle (2008)) and has many advantages such as low costs, flexibility, reliability, quality \& security through their communities, freedom, and transparency (Odun-Ayo et al. (2018), Morgan and Finnegan (2007)). On the other 
Table 1: List of popular Open-Source Software (OSS) usable towards executing specific tasks or applications.

\begin{tabular}{|l|l|l|}
\hline Application & Description & Example of OSS \\
\hline Relational Databases & $\begin{array}{l}\text { Database type used for storing and reading } \\
\text { data with a known schema. Data is queried } \\
\text { through SQL }\end{array}$ & $\begin{array}{l}\text { MySQL, PostgreSQL and } \\
\text { SQLite }\end{array}$ \\
\hline Containerization & $\begin{array}{l}\text { Containers are used to deploy packaged ap- } \\
\text { plications locally or in the cloud }\end{array}$ & Docker and Kuberneties \\
\hline Data visualization & $\begin{array}{l}\text { Libraries used to plot and visualize data. } \\
\text { They can offer support for various program- } \\
\text { ming languages via interfaces }\end{array}$ & D3 and Plotly \\
\hline Distributed SQL Query Engines & $\begin{array}{l}\text { Software that enables fast query of heteroge- } \\
\text { nous data and very large data sets (TB+) }\end{array}$ & Hive, Presto and Spark \\
\hline File Formats & Data containers based on files & Avro, CSV and Parquet \\
\hline Web Frameworks* & $\begin{array}{l}\text { Frameworks enabling rapid development of } \\
\text { web interfaces. }\end{array}$ & Django and Flask \\
\hline
\end{tabular}

*This paper focuses on Python but many frameworks are available in others languages.

hand, there are drawbacks to OSS such as lack of extensive support or not being as user-friendly as commercial solutions (Morgan and Finnegan (2007)).

OSS can be found in various applications. Table 1 offers a listing of popular OSS that are used in the cloud industry for a specific application such as data storage.

\section{BIS Case Study}

Through this Building Information System case study, we will dive into three steps of the whole infrastructure: data collection and storage, simulation environment and finally User Interface and visualization.

\section{Data Collection and Storage}

Sharing raw data generated by a BAS has been challenging due to proprietary protocols and security considerations. This issue is mitigated by the growing adoptions of the BACnet protocol, developed by the American Society of Heating, Refrigerating and AirConditioning Engineers (ASHRAE) in the 1990s and now an ISO standard. Additionally its use over compatible data link and physical layers such as IPv4 over Ethernet allows fast setup of Data Acquisition Units (DAUs) in conjunction with OSS such as VOLTTRON. DAUs enable Data Acquisition (DAQ) at regular intervals (5-min interval for most cases) and sharing data to a cloud storage solution through a secured connection. IT security considerations are still present which can require the use of VPNs or proxies. A cloud storage solution, known as Facts Service, has been developed using the Python programming language, the Flask micro web framework to handle HTTPS requests and the PostgreSQL relational database (PostgreSQL Global Development Group (2019)) to store metadata. The Python code and its dependencies are packaged in a Docker container to enable fast and consistent deployment on the cloud platform.
Simulation often relies on files to store data such as CSVs. Querying data is not easy unless the entire dataset is stored into memory, making it impractical for large datasets. Moving to a cloud relational database enabled remote and consistent access to data. Using SQL, very large amount of data is queryable quickly and efficiently. However as the dataset kept growing so were the costs. A solution was to move back to files using the open-source columnar data storage format known as Apache Parquet (Apache Software Foundation (2019)). Parquet supports very efficient compression allowing small file sizes compared to CSV or PostgreSQL table as demonstrated in Figure 3. These files can then be stored on key-object stores such as Amazon's S3 at one fifth the cost of cloud database storage. Combined with distributed SQL query engines such as Presto, data can still be queried using SQL while keeping the cost down. This approach gives the best of both worlds with minimized downsides such as consistent reads.

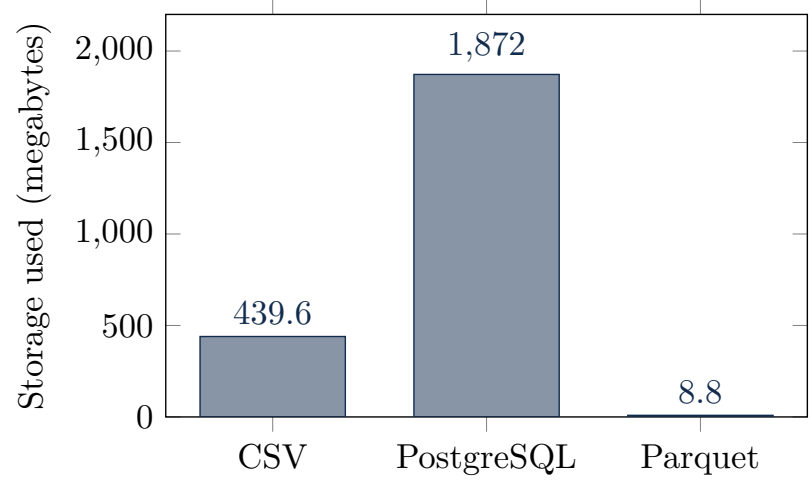

Figure 3: Storage utilization comparison of 2 data file formats and 1 database table format: Comma Separated Values (CSV), Parquet and PostgreSQL table. All stores contain 14818630 records of building data consisting each of an id, a timestamp and a float. Parquet file format offers in this example a $50 x \mathrm{com}$ pression ratio against the CSV file format. 
Table 2: Pros and Cons of different data storage solutions.

\begin{tabular}{|c|c|c|c|}
\hline & CSV & PostgreSQL & Presto with Parquet \\
\hline Pros & $\begin{array}{l}\text { - Great read performance } \\
\text { (especially on small datasets) } \\
\text { - Available as export format } \\
\text { of many software }\end{array}$ & $\begin{array}{l}\text { - Query language built-in } \\
\text { - Fast lookup of data within } \\
\text { table (SELECT operation) } \\
\text { - Consistent data access speed }\end{array}$ & $\begin{array}{l}\text { - Query language built-in } \\
\text { - Small dataset storage footprint } \\
\text { - Distributed system handling } \\
\text { large datasets }\end{array}$ \\
\hline Cons & $\begin{array}{l}\text { - External libraries needed to } \\
\text { support queries } \\
\text { - Files have to be loaded into } \\
\text { memory to be queryable }\end{array}$ & $\begin{array}{l}\text { - Expensive due to large } \\
\text { dataset storage footprint }\end{array}$ & $\begin{array}{l}\text { - Inconsistent data access speed } \\
\text { - Slow access on small datasets }\end{array}$ \\
\hline
\end{tabular}

Most CSPs offer fully managed instances for databases, map-reduce clusters or key-object stores allowing the developer to focus on using these cloud technologies while not having to worry about maintenance nor setup.

Each storage solution offers its own set of pros and cons as shown in Table 2. CSV files are easy to work with and implement since they are the de-facto export format for data in many software solutions. However, due to their underlying nature, they do not offer a native query language nor support for various compression schemes. PostgreSQL and other relational databases offer often fast lookup of data due to the use of indexes, are consistent at the expense of being very costly to operate with large amounts of data. Finally Presto using the Parquet file format, offers low operation costs due to its distributed nature and compressed dataset but reads are not consistent which can be an issue for visualization and UI.



Figure 4: Time to select a month (14 818630 records), a day (462 119 records) within a month and a day of building data using CSV, PostgreSQL and Presto with Parquet on the same instance type ( $A W S$ m4.large, 2 virtual CPUs and 8 GB of RAM).

Overall, distributed SQL query engines should be reserved for processing of large datasets and simulation, relational databases for storage of results meant to be visualized rapidly, and CSV files for prototyping on small datasets. As shown in Figure 4, the time to query building data can widely vary depending on the task at hand such as accessing large and small datasets or querying a subset of data within a larger dataset.

\section{Simulation environment}

Analysis of time-series building performance data is executed using an internal simulation environment known as PyRithm. PyRithm is a software for AFDD. The environment is based on analysis and modeling techniques previously presented in Georgescu et al. (2017), Nesztler et al. (2016) and Littooy et al. (2016). Essentially, sensors from a real or simulated building are modeled against a metadata schema (Balaji et al. (2016)) enabling common descriptors to be applied across a wide range of building HVAC system hierarchies. These descriptors enable a system of equations to be realized that describes diagnostics of the simulated building system. These diagnostics are applied based on the metadata schema types so diagnostics are not equipment or system type specific, but are agglomerative based on the measurements available on a simulated equipment or device. Measurements used include data from actuators (e.g. dampers, heating / cooling coils), sensors (e.g. temperature, pressure, flows), and control system signals (e.g. status / modes, schedules, alarms).

The execution of simulations is orchestrated using Docker containers. The source code of the software, and its dependencies, are stored in an image, and for each simulation, a container is instantiated. An advantage of this workflow is that it enables the application to be executed in a computational environment that is portable to other machines also running the container engine. In a numerical computing setting, this approach offers the benefit to practitioners of being able to reproduce the runtime of applications (or simulations) to any other computer running the container engine. Thus, experiments can be developed on one's local machine and distributed to other researchers or executed in other environments that have more powerful computational resources.

Even if cloud platforms enable scalable infrastructures that local ones often can't match, some individual tasks can have quicker runtimes locally due to the differences in the underlying hardware infrastructure such as CPU or RAM clock speeds, networking or microarchitectures. Figure 5 shows that the same 
Docker container simulation task running locally on a laptop can execute faster than on the cloud using the equivalent allocated resources (2 virtual CPUs and 4 GB of RAM).

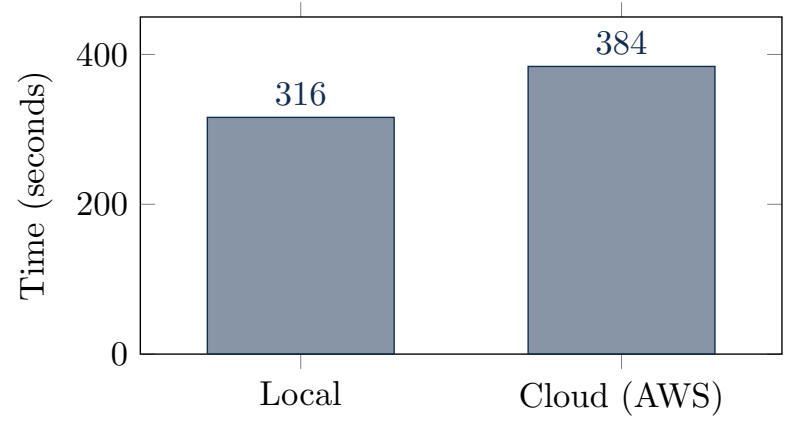

Figure 5: Runtime of building simulation software for a day of building data running locally and on the Cloud (AWS t3.medium) using Docker containers with 2 virtual $C P U s$ and $4 G B$ of RAM allocated.

\section{User Interface and Visualization}

When running software in the cloud, multiple interfaces are available to interact with it such as an User Interface (UI) or a Command Line Interface (CLI). However to reduce the barrier of entry, an UI is better suited due to its ergonomics. This interface needs also to be accessible remotely on as much platforms as possible. A solution resides in designing a website mimicking a local application, allowing anyone with an Internet connection to do work through a Web browser.

A web interface was designed to support many aspects of building simulations such as raw data and simulation arguments management; raw data and results visualization; and simulation remote execution and scheduling through an ergonomic UI with tailored micro-services.

Coming from a data scientist and engineer background, the development of this interface, internally known as Platform, needed to use as many tools as possible known by all parties. That is why it was developed in Python using the open-source and popular Django web framework.

Data visualization is handled with tables and/or Web plotting libraries such as Plotly, D3 or Chart.js allowing support for a wide variety of visualizations. Also libraries such as Plotly can provide interfaces in many languages such as Python, Matlab or R allowing a smooth transition between existing workflows and web interfaces.

Overall, a web interface creates a collaborative work environment where anyone involved in a project can bring their own expertise. It can bring together existing processes and workflows together enabling the use of the application from anywhere in the world. For researchers, it offers the ability to easily share work and results in an efficient manner while being accessible to other researchers for validation and transparency purposes. Moreover, a web application in combination with containers can enable a very high degree of reproducibility of the work since all simulation parameters can be accessible within the interface. This reproducibility can help the community move towards a clean research initiative encouraging researchers becoming better scientists.

Finally, cloud technologies are often based on open standards enabling interoperability between current and future software making the time developing the simulation software a sound investment.

\section{Conclusion}

In this paper, frameworks, services, and the offerings of Cloud Service Providers (CSPs) are described as they relate to developing a software stack which can utilize these technologies for efficient compute, storage, and visualization or UI.

These tools can enable software architectures for the building simulation community in that they (1) enable work to be reproducible in different computing environments thus allowing work to be more distributable to other researchers, (2) allow scalability in that additional resources can be provisioned as a needed, (3) are, on a per unit work basis, often less expensive compared to a locally managed environment. The goal of this paper is to raise a discourse on how these technologies can be implemented in the building simulation community. A practitioner doesn't need to apply all techniques described to realize some benefit. In many cases, only small changes need to be made. The following best-practices can be applied to many projects with minimal modification of existing applications: (1) the use of Docker/containerization to decouple their application from specific configuration of the host machine. (2) the adoption of D3, Plotly, or some other Javascript based visualization framework so that figures and graphs are HTML-native and thus compatible across multiple platforms.

With these recommendations, there is of course a balance to maintain between an application being distributable, ease of development, and the lifespan of a project. These considerations exist on a project by project basis. It is foreseeable though that, as cloud managed services become more refined, and technologies such as IoT become more ubiquitous, the adoption of cloud technologies may be more of a requirement. A great potential of CSPs is that they provide a platform to work with building simulations, virtual devices, and physical infrastructure/devices within a single context.

\section{Acknowledgments}

The authors would like to thank the developers of open-source software who continuously help grow and support the community. 


\section{References}

Apache Software Foundation (2019). Apache Parquet. [Online; accessed 1 January 2019].

American Society of Heating, Refrigerating and AirConditioning Engineers (2016). BACnet-A Data Communication Protocol for Building Automation and Control Networks.

American Society of Heating, Refrigerating and AirConditioning Engineers (2018). Standard for Commercial Building Energy Audits (ASHRAE 2112018).

Balaji, B., A. Bhattacharya, G. Fierro, J. Gao, J. Gluck, D. Hong, A. Johansen, J. Koh, J. Ploennigs, Y. Agarwal, M. Berges, D. Culler, R. Gupta, M. B. Kjærgaard, M. Srivastava, and K. Whitehouse (2016). Brick: Towards a unified metadata schema for buildings. In Proceedings of the 3rd ACM International Conference on Systems for Energy-Efficient Built Environments, BuildSys '16, New York, NY, USA, pp. 41-50. ACM.

Basarkar, M., X. Pang, L. Wang, P. Haves, and T. Hong (2011). Modeling and simulation of hvac faults in energyplus. In Proceedings of Building Simulation 2011: 12th Conference of International Building Performance Simulation Association. Sydney (Australia), 14-16 November 2011.

Deshpande, A. and D. Riehle (2008). The total growth of open source. In B. Russo, E. Damiani, S. Hissam, B. Lundell, and G. Succi (Eds), Open Source Development, Communities and Quality, Boston, MA, pp. 197-209. Springer US.

Djuric, N. and V. Novakovic (2009, February). Review of possibilities and necessities for building lifetime commissioning. Renewable and Sustainable Energy Reviews 13(2), 486-492.

Georgescu, M., S. Loire, D. Kasper, and I. Mezic (2017). Whole-building fault detection: A scalable approach using spectral methods. In Proceedings of the 2017 ASHRAE Winter Meeting. Las Vegas (NV, USA).

International Organization for Standardization (2014). Information technology - Cloud computing - Overview and vocabulary (ISO 177888).

Littooy, B., S. Loire, M. Georgescu, and I. Mezić (2016, Dec). Pattern recognition and classification of HVAC rule-based faults in commercial buildings. In 2016 IEEE International Conference on Big Data (Big Data), pp. 1412-1421.

Martin, R. C. (2017). Clean Architecture: A Craftsman's Guide to Software Structure and Design. Robert C. Martin Series. Boston, MA: Prentice Hall.
Morgan, L. and P. Finnegan (2007). Benefits and drawbacks of open source software: An exploratory study of secondary software firms. In J. Feller, B. Fitzgerald, W. Scacchi, and A. Sillitti (Eds), Open Source Development, Adoption and Innovation, Boston, MA, pp. 307-312. Springer US.

Nesztler, T., D. Kasper, M. Georgescu, S. Loire, and I. Mezić (2016, Dec). Uniformization, organization, association and use of metadata from multiple content providers and manufacturers: A close look at the Building Automation System (BAS) sector. In 2016 IEEE International Conference on Big Data (Big Data), pp. 1633-1638.

National Institute of Standards and Technology (2011). Guide to Security for Full Virtualization Technologies (NIST SP 800-125).

Odun-Ayo, I., A. Falade, and V. Samuel (2018). Cloud computing and open source software: Issues and developments. In Proceedings of the International MultiConference of Engineers and Computer Scientists 2018 Vol I. Hong Kong, 14-16 March 2018.

Open Source Initiative (2019). The open source definition. [Online; accessed 1 January 2019].

PostgreSQL Global Development Group (2019). PostgreSQL. [Online; accessed 1 January 2019].

Project Haystack Corporation (2019). Project Haystack. [Online; accessed 1 January 2019].

Richman, R., H. Zirnhelt, and S. Fix (2014). Largescale building simulation using cloud computing for estimating lifecycle energy consumption. Canadian Journal of Civil Engineering 41(3), 252-262.

Torcellini, P., S. Pless, M. Deru, and D. Crawley (2006). Zero energy buildings: A critical look at the definition. In ACEEE Summer Study. Pacific Grove, CA (USA), August 14-18, 2006.

U.S. Department of Energy (2012). 2011 Buildings Energy Data Book. U.S. Department of Energy.

VOLTTRON (2019). VOLTTRON. [Online; accessed 1 January 2019].

Wang, L., S. Greenberg, J. Fiegel, A. Rubalcava, S. Earni, X. Pang, R. Yin, S. Woodworth, and J. Hernandez-Maldonado (2013). Monitoring-based hvac commissioning of an existing office building for energy efficiency. Applied Energy 102(C), 13821390.

Wetter, M. (2011). Co-simulation of building energy and control systems with the building controls virtual test bed. Journal of Building Performance Simulation 4(3), 185-203. 